\title{
Attosecond photoionization dynamics in the vicinity of the Cooper minima in argon
}

\author{
C. Alexandridi, ${ }^{1,}{ }^{*}$ D. Platzer, ${ }^{1,}{ }^{*}$ L. Barreau $\odot,{ }^{1}$ D. Busto $\odot,{ }^{2}$ S. Zhong $\odot,{ }^{2}$ M. Turconi, ${ }^{1}$ L. Neoričić $\odot,{ }^{2}$ H. Laurell $\odot,{ }^{2}$ \\ C. L. Arnold, ${ }^{2}$ A. Borot, ${ }^{1}$ J.-F. Hergott, ${ }^{1}$ O. Tcherbakoff, ${ }^{1}$ M. Lejman ${ }^{\circledR},{ }^{1}$ M. Gisselbrecht $\odot,{ }^{2}$ E. Lindroth $\odot,{ }^{3}$ A. L'Huillier $\odot,{ }^{2}$ \\ J. M. Dahlström, ${ }^{2}$ and P. Salières (ib ${ }^{1}$ \\ ${ }^{1}$ Université Paris-Saclay, CEA, CNRS, LIDYL, 91191 Gif-sur-Yvette, France \\ ${ }^{2}$ Department of Physics, Lund University, Box 118, SE-22100 Lund, Sweden \\ ${ }^{3}$ Department of Physics, Stockholm University, AlbaNova University Center, SE-10691 Stockholm, Sweden
}

(Received 29 July 2020; accepted 22 December 2020; published 5 February 2021)

\begin{abstract}
Using a spectrally resolved electron interferometry technique, we measure photoionization time delays between the $3 s$ and $3 p$ subshells of argon over a large 34-eV energy range covering the Cooper minima in both subshells. The observed strong variations of the $3 s-3 p$ delay difference, including a sign change, are well reproduced by theoretical calculations using the two-photon two-color random-phase approximation with exchange. Strong shake-up channels lead to photoelectrons spectrally overlapping with those emitted from the $3 s$ subshell. These channels need to be included in our analysis to reproduce the experimental data. Our measurements provide a benchmark for multielectronic theoretical models aiming at an accurate description of interchannel correlation.
\end{abstract}

DOI: 10.1103/PhysRevResearch.3.L012012

Half a century after their theoretical description by Eisenbud [1], Wigner [2], and Smith [3], scattering delays-also called Wigner delays - can now be measured using attosecond spectroscopy, which allows for detailed studies of the correlated interactions within various quantum systems. In practice, this is done by recording the spectral variation of the quantum phase of electron wave packets photoemitted from solids [4,5], molecules [6-9], or atoms [10-21]. Of particular interest in scattering physics are spectral structures in the continua of atoms and molecules such as autoionizing resonances [22,23], shape resonances [24], and Cooper minima [25], as they carry detailed information on the internal structure, electronic correlations, potential, and orbital shapes. The rapidly varying phase in the vicinity of these structures has recently been investigated, e.g., for autoionizing [26-30] and shape resonances [7]. As for Cooper minima (CM), very few measurements have been performed, either in photoionization [14] or photorecombination [31,32] spectroscopy.

Ionization of argon from the $n=3$ shell has attracted considerable attention due to abundant signatures of intraand interorbital electronic correlations. In the case of the $3 p$ subshell, intraorbital correlation is important close to the ionization threshold, due to the so-called ground-state correlation [33]. At higher photon energies, where the photoionization process can be described using single active electron models, the sign change in the $3 p \rightarrow \varepsilon d$ radial transition matrix element leads to a CM close to $53 \mathrm{eV}[25,34]$. As for the

\footnotetext{
${ }^{*}$ These authors contributed equally to this work.
}

Published by the American Physical Society under the terms of the Creative Commons Attribution 4.0 International license. Further distribution of this work must maintain attribution to the author(s) and the published article's title, journal citation, and DOI.
$3 s$ subshell, interorbital correlation is important, since the $3 p \rightarrow \varepsilon d$ process is strongly coupled to $3 s \rightarrow \varepsilon p$ [35]. This leads to a correlation-induced "replica" of the $3 p \mathrm{CM}$ in the $3 s$ ionization channel, close to $42-\mathrm{eV}$ photon energy, as shown in Fig. 1 [36].

All these correlation effects are expected to leave an imprint on the scattering and photoionization delays between the $3 s$ and $3 p$ electrons, which motivated a large number of calculations during the past decade in the demanding region above the $3 s$ threshold [11,12,37-45]. The methods qualitatively agree on the behavior of the $3 p$ atomic delays, which are slightly negative over a large energy region around the $3 p$ $\mathrm{CM}$. However, for the $3 s$ case, the atomic delays close to the $3 s \mathrm{CM}$ strongly differ in magnitude and in sign depending on the degree of correlation included $[39,44]$. Up to now, two experiments using the RABBIT (reconstruction of attosecond beating by interference of two-photon transitions) technique have aimed at measuring the photoionization time-delay difference in the $n=3$ shell of argon [11,12]. Unfortunately, for experimental reasons, the results were limited to the 34- to 40$\mathrm{eV}$ photon energy range, below the $3 s$ and $3 p \mathrm{CM}$, preventing a detailed comparison over the entire spectral region.

In this Letter, we measure photoionization time delays between the $3 s$ and $3 p$ subshells of argon over a large energy range (34-68 eV) covering the $\mathrm{CM}$ in both subshells. The presence of multiple ionization channels leads to spectral congestion, which is one of the main experimental challenges. The spectrally resolved interferometric Rainbow RABBIT technique $[27,28]$ allows us to substantially overcome this difficulty. The experimental results show strong variations of the $3 s-3 p$ delay difference, with a change of sign in the $3 s \mathrm{CM}$ region. They are compared to theoretical calculations using the two-photon two-color random-phase approximation with exchange (2P2C-RPAE) method [44]. The agreement is excellent in-and above-the $3 p \mathrm{CM}$ region $(45-68 \mathrm{eV})$ and 


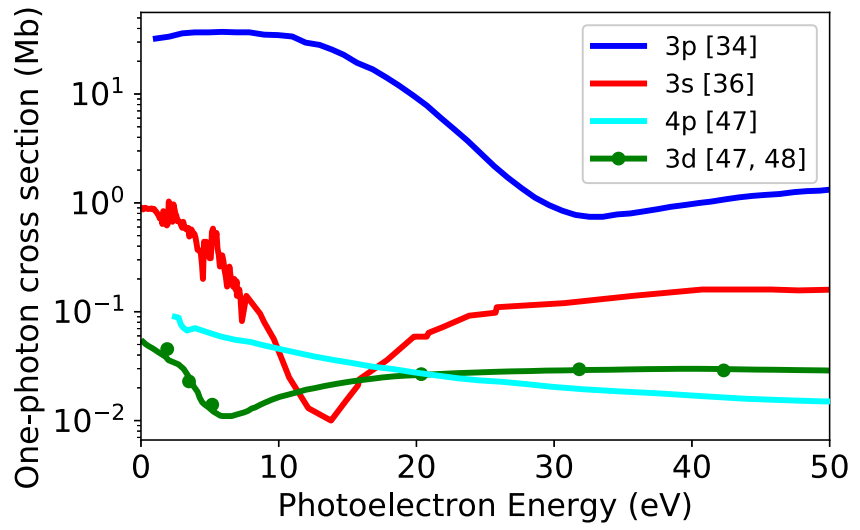

FIG. 1. One-photon ionization cross sections of the $3 p$ (blue/black) and $3 s$ (red/medium gray) channels [34,36], together with that of the satellite shake-up states $3 s^{2} 3 p^{4}\left({ }^{1} D\right) 4 p\left({ }^{2} P\right)$ (cyan/light gray) [47] and $3 s^{2} 3 p^{4}\left({ }^{1} D\right) 3 d\left({ }^{2} S\right)$ (green/dark gray dots for experiments [48] and solid line for theory [47]) labeled $4 p$ and $3 d$, respectively. The ionization thresholds for $3 p, 3 s, 4 p$, and $3 d$ are $15.76,29.24,37.15$, and $38.60 \mathrm{eV}$, respectively.

unsatisfactory in the $3 s \mathrm{CM}$ region $(34-45 \mathrm{eV})$. We show that the presence of electrons from strong shake-up (SU) channels overlapping with $3 s$ electrons is the likely reason for this deviation. By taking into account the dominant SU contribution, a good agreement between theory and experiment can be obtained in the 34- to $39-\mathrm{eV}$ range.

The experiments were performed independently at the ATTOLab facility in Saclay, France, and at Lund University, Sweden. The details of the two setups are described in the Supplemental Material [46]. Briefly, intense infrared (IR) femtosecond pulses are split in a Mach-Zehnder interferometer. In one arm of the interferometer, high-order harmonics are generated in neon gas and spectrally filtered by metallic foils. In the other arm, a small fraction of the IR radiation is temporally delayed. Both beams are recombined and then focused into an argon gas jet. The emitted electrons are detected with a 2-m-long magnetic bottle electron spectrometer (MBES).

To measure the photoionization time delays, we used the Rainbow RABBIT technique [27] which is schematically illustrated in Fig. 2(a). A comb of coherent harmonics ionizes argon atoms, creating one-photon electron wave packets (EWPs) from both $3 s$ and $3 p$ subshells. The corresponding electron peaks are referred to as " $3 s$ or $3 p$ harmonics" in the following. By adding the weak IR dressing field $\left(\sim 10^{11} \mathrm{~W} / \mathrm{cm}^{2}\right)$, replicas of the initial EWPs are created by two-photon XUV \pm IR transitions to the same final states. Their interference gives rise to the so-called sidebands (SB), the intensity of which oscillates as a function of the delay $\tau$ between the XUV and IR pulses as

$$
S B_{n, i}(\tau)=A_{n, i}+B_{n, i} \cos \left[2 \omega_{0} \tau-\Delta \phi_{n}^{\mathrm{XUV}}-\Delta \phi_{n, i}^{\mathrm{A}}\right],
$$

where $i$ is the ionization channel $\left(3 s, 3 p\right.$, etc.), $\omega_{0}$ is the angular frequency of the driving laser, $\Delta \phi_{n}^{\mathrm{XUV}}=\phi_{n+1}-\phi_{n-1}$ is the phase difference between two consecutive harmonics with orders $n \pm 1$ [49], and $\Delta \phi_{n, i}^{\mathrm{A}}$ is the phase difference between the two-photon transition dipole matrix elements. In the so-called asymptotic approximation [50], $\Delta \phi_{n, i}^{\mathrm{A}}$ can be ex-

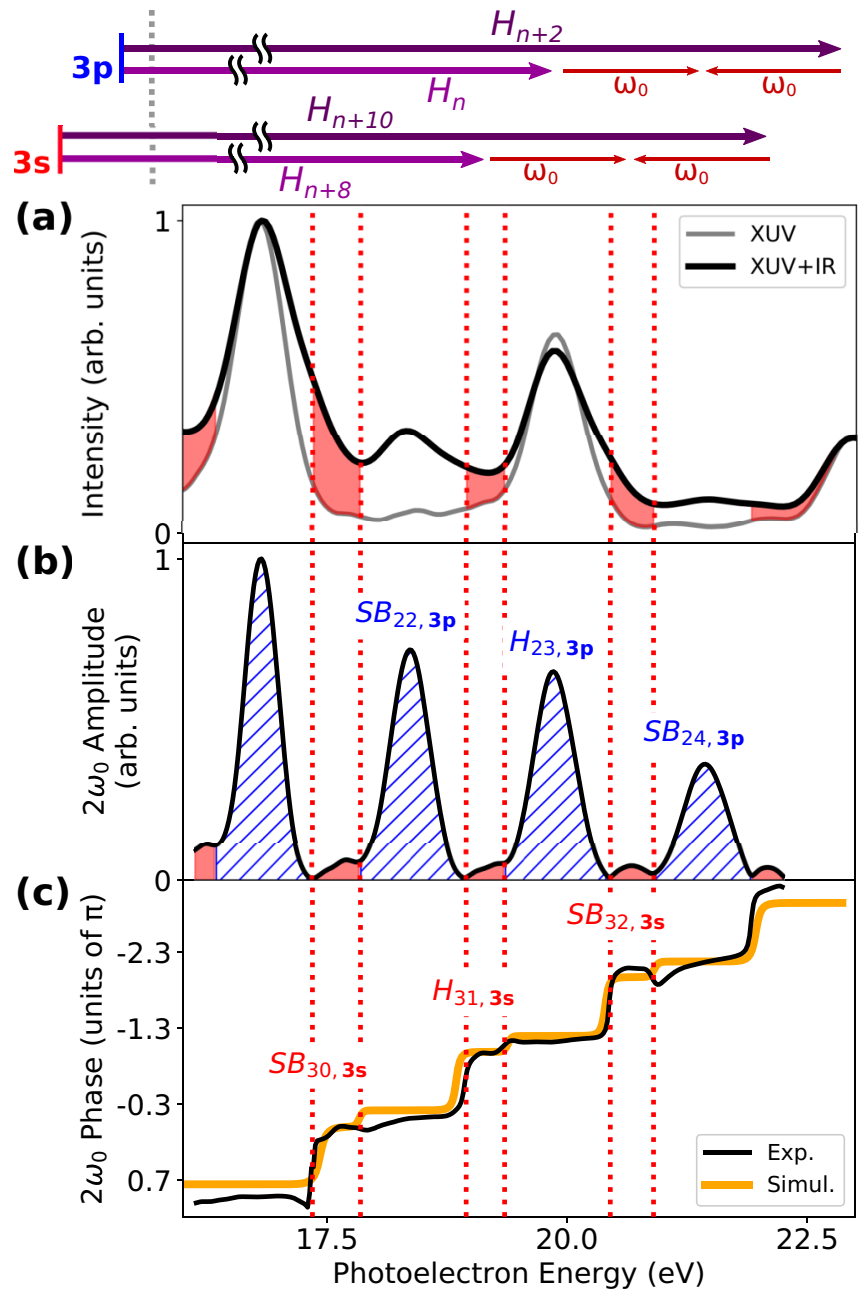

FIG. 2. (a) XUV + IR photoelectron spectrum (black), obtained in Saclay by integrating the RABBIT spectrogram over the delay, as compared to the XUV-only spectrum (gray), and corresponding multiphoton transitions for the $3 s$ and $3 p$ channels. Spectral amplitude (b) and phase (c) of the $2 \omega_{0}$ oscillations obtained from a Fourier transform at each energy of the RABBIT spectrogram. In panel (b), the amplitudes related to the $3 p(3 s)$ channel are highlighted in hatched blue (filled red), respectively. The red dashed lines indicate the spectral regions with dominant $3 s$ contributions. In panel (c), the measured phase evolution (black line) is compared to the simulated one (orange/gray line) [46].

pressed as the sum of two contributions, $\Delta \eta_{n, i}+\Delta \phi_{n, i}^{\mathrm{cc}}$, where $\eta_{n \pm 1, i}$ is the scattering phase accumulated by the EWP in the one-photon (XUV) transition, which is intrinsic to the target atom, and $\phi_{n \pm 1, i}^{\mathrm{cc}}$ is a quasiuniversal measurement-induced phase shift due to the electron being probed by the IR laser field in a long-range potential with a Coulomb tail [50].

Group delays can be defined through $\tau \approx \Delta \phi / 2 \omega_{0}$. The measured delay can be expressed as $\tau_{n}^{\mathrm{XUV}}+\tau_{n, i}^{\mathrm{A}}$. Introducing the Wigner delay, $\tau_{n, i}^{\mathrm{W}} \approx \Delta \eta_{n, i} / 2 \omega_{0}$ and $\tau_{n, i}^{\mathrm{A}} \approx \tau_{n, i}^{\mathrm{W}}+\tau_{n, i}^{\mathrm{cc}}$. Since the $3 s$ and $3 p$ photoelectrons are ionized by the same harmonic comb, the $\tau_{n}^{\mathrm{XUV}}$ contribution of the ionizing radiation can be removed by calculating the difference of the delays for the two channels, giving direct access to $\tau_{3 s}^{\mathrm{A}}-\tau_{3 p}^{\mathrm{A}}$ with high accuracy. 
Two main experimental difficulties have prevented the measurement of this delay difference over the whole CM region. The first is that the ionization cross section is much weaker for $3 s$ than for $3 p$, as shown in Fig. 1. The second is the spectral overlap of the two channels: the $3 s$ sidebands $S B_{n, 3 s}$ fall only $0.47 \mathrm{eV}$ from the much more intense $3 p$ harmonics $H_{n-9,3 p}$ due to the difference in ionization energies $\Delta E_{3 s-3 p}=$ $13.48 \mathrm{eV}=9 \hbar \omega_{0}-0.47 \mathrm{eV}$. To cope with these difficulties, previous studies $[11,12]$ spectrally isolated the two contributions by photoionizing with only four harmonics $\left(H_{21}-H_{27}\right)$, selected by using a combination of filters and generation in argon. The main drawback of this approach is that it constrains the usable energy range to a $10-\mathrm{eV}$ window below $40 \mathrm{eV}$.

In the present work, harmonics generated in neon were used as the ionizing radiation. Neon has a generation efficiency smaller than that of argon, but exhibits a quite flat harmonic spectrum with a much higher cutoff energy. In combination with a single 200-nm-thick $\mathrm{Al}$ filter, a broad spectrum (20-72 eV) including harmonics $H_{13}-H_{45}$ is obtained. Under these conditions, the $3 p$ harmonics overshadow the $3 s$ sidebands. In Fig. 2(a), the contributions of the $3 s$ SBs (harmonics) in the XUV + IR spectrum appear as small shoulders highlighted on the blue side of the $3 p$ harmonics (SBs), as evidenced by the comparison with the XUV-only spectrum. In order to separate the two contributions, we perform a Rainbow RABBIT analysis of the recorded spectrogram; i.e., we analyze the $2 \omega_{0}$ oscillations for each energy $E$ in the spectrum [see Eq. (1)]. The amplitude and the phase of these oscillations are shown in Figs. 2(b) and 2(c), respectively.

Between consecutive harmonics and sidebands of the same ionization channel, a dephasing of $\simeq \pi$ is expected, as a result of the conservation of the total number of electrons [46]. Figure 2(c) shows multiple phase jumps, some of them close to $\pi$, others much less than $\pi$, which occur between sidebands (or harmonics) of different subshells. In addition to the high resolution of the MBES, the combination of amplitude and phase measurements allows us to distinguish the $3 s$ contribution from the strong neighboring $3 p$ electron peaks (see the dotted lines).

The measured $\tau_{3 s}^{\mathrm{A}}-\tau_{3 p}^{\mathrm{A}}$ delay differences are plotted in Fig. 3(a). The results reveal an interesting feature, namely, a change of sign around $43 \mathrm{eV}$. The negative sign in the lowenergy region (close to the $3 s$ threshold) is in part explained by the large negative delay contribution due to continuumcontinuum transitions of slow photoelectrons [51]. However, in neon, the atomic delay difference between the $2 s$ and $2 p$ electrons stays negative up to high $\sim 100-\mathrm{eV}$ energies [19]. The positive sign in argon is thus a probable signature of the CM. Finally, the delay difference converges towards zero for higher energies where the $3 s$ and $3 p$ cross sections are unstructured.

The low-energy region is a particularly difficult range for the measurements because of the very weak signal of the $3 \mathrm{~s}$ channel due to the $\mathrm{CM}$, resulting in more than 3 orders of magnitude difference with the $3 p$ channel [see Fig. 1(a)]. An extended analysis over a larger set of data was thus performed to improve the statistical significance of the result in the 34- to $43-\mathrm{eV}$ spectral range corresponding to orders 22 to 27 . There, the phases were extracted for both sideband and harmonic peaks. As discussed in Fig. 2(b), the harmonic phases are

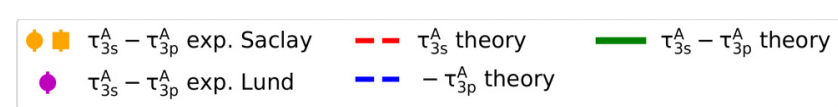

(a)
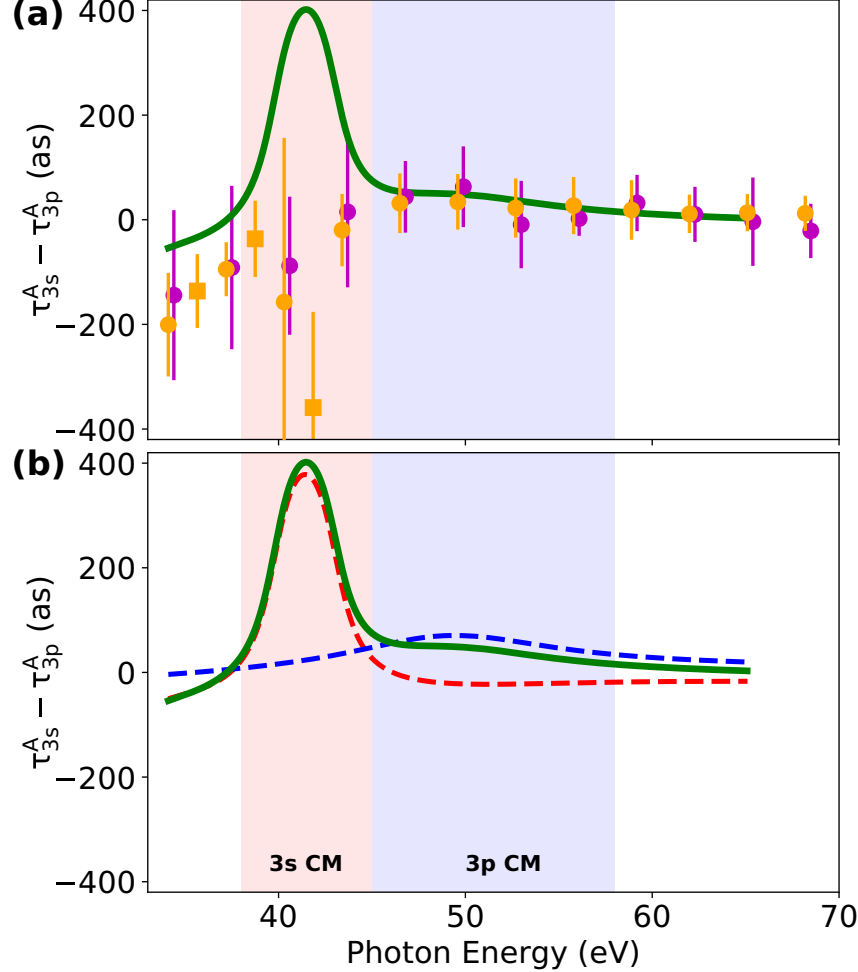

FIG. 3. Atomic delay difference between $3 s$ and $3 p$ ionization channels in the vicinity of the $3 s \mathrm{CM}$ and $3 p \mathrm{CM}$ (highlighted in red/medium gray and blue/dark gray, respectively). (a) Experimental data from Lund (magenta/dark gray dots) and Saclay (orange/light gray markers, circles for sidebands, squares for harmonics) compared with simulations using the 2P2C-RPAE model (green/dark gray line). (b) Decomposition of the simulated delaydifference curve (green/dark gray line) into the $3 \mathrm{~s}$ (red/gray dashed line) and $3 p$ (blue/black dashed line) delay curves.

shifted by $\sim \pi$ relative to the SBs. However, this $\pi$ phase shift cancels when we calculate the delay difference between $3 s$ and $3 p$ harmonics. As shown in Fig. 3(a), the harmonic delays behave as an average of the neighboring sideband delays [46], with the only exception being $H_{27}$ at $42 \mathrm{eV}$.

In Fig. 3, the experimental results are compared with theoretical predictions using the recently developed $2 \mathrm{P} 2 \mathrm{C}$ RPAE method [44]. It consists of calculating complete self-consistent two-photon processes, including electron correlation in bound-continuum and continuum-continuum transitions, ion polarization effects, and the reversed photon time orders. The orbital energies of $3 p$ and $3 s$ are adjusted to fit the experimental ionization thresholds and the interaction with the fields is computed in the length gauge. The calculated $\tau_{3 p}^{\mathrm{A}}$ and $\tau_{3 s}^{\mathrm{A}}$ include the contribution from all emission angles, which corresponds to our experimental configurations. For $\tau_{3 p}^{\mathrm{A}}$, this integration significantly modifies the delays as compared to that in the XUV polarization direction [14,39], while for $\tau_{3 s}^{\mathrm{A}}$ little effect is observed due to the single $3 s \rightarrow \varepsilon p$ transition. Earlier work [39] shows that the full one-photon RPAE theory yields a $3 s \mathrm{CM}$ at $40 \mathrm{eV}$, while the restricted one-photon RPAE 
calculation with only intrashell $(n=3)$ correlation produces a $\mathrm{CM}$ close to $42 \mathrm{eV}$ in better agreement with one-photon cross section measurements [36]. For this reason, we use the latter method for the $3 s$ 2P2C-RPAE simulations in Fig. 3(b), which moves the positive peak of $\tau_{3 s}^{\mathrm{A}}$ from $40 \mathrm{eV}$ to the correct position of $42 \mathrm{eV} \mathrm{[46].}$

An excellent agreement between the simulations and the experimental data is observed in the $3 p \mathrm{CM}$ region and above (45- to 68-eV range), as shown in Fig. 3(a). For the lower energies, a small deviation in the 34-to 39-eV range and a disagreement in the 39- to $42-\mathrm{eV}$ range can be noticed. Note that our measurements are fully consistent with earlier experimental results in the 34- to $40-\mathrm{eV}$ range [11,12].

We attribute these differences to the presence of unresolved additional ionic channels that perturb the measurements. In this $3 s \mathrm{CM}$ region where the cross section drops, two SU channels associated with the $3 s^{2} 3 p^{4}\left({ }^{1} D\right) 3 d\left({ }^{2} S\right)$ and $3 s^{2} 3 p^{4}\left({ }^{1} D\right) 4 p\left({ }^{2} P\right)$ ionic states, hereafter denoted $3 d$ and $4 p$ channels, give significant contributions to the photoelectron spectrum and may even dominate over $3 s$, as shown in Fig. 1. The $4 p$ channel has $H_{n+5,4 p}$ overlapping with $S B_{n, 3 s}$ due to the difference in ion excitation energies being five times the laser photon energy, $\Delta E_{4 p-3 s}=7.90 \mathrm{eV} \approx 5 \hbar \omega_{0}$. Similarly, the $3 d$ channel has $S B_{n+6,3 d}$ overlapping with $S B_{n, 3 s}$, due to $\Delta E_{3 d-3 s}=9.34 \mathrm{eV} \approx 6 \hbar \omega_{0}$.

We first consider the $34-$ to $39-\mathrm{eV}$ range, where $3 s$ and $4 p$ are the dominant channels. We model the RABBIT modulation amplitude for a given channel by assuming that it is proportional to the one-photon cross section at a given final kinetic energy of the photoelectron [46]. This allows us to make simple estimates of the modulation strengths $B_{n, i}$ in Eq. (1) assuming constant harmonic photon flux [see Fig. 4(a)]. The crossing between the $3 s$ and $4 p$ modulation strengths close to $39 \mathrm{eV}$ results in a significant $\mathrm{SU}$ contamination of the $3 \mathrm{~s}$ time-delay measurements in this energy range.

Due to their different symmetries, the $3 s$ and $4 p$ channels add incoherently in this angular-integrated measurement [52]. The incoherent sum of the two signals, $S B_{n, 3 s}$ and $H_{n+5,4 p}$, then results in a phase-shifted and amplitude-modified oscillating function, $B_{t} \cos \left(2 \omega_{0} \tau-\theta_{t}\right)$, where

$$
\begin{aligned}
& \theta_{t}=\tan ^{-1}\left(\frac{\sin \left(\delta_{n}\right)}{B_{n, 3 s} / B_{n+5,4 p}+\cos \left(\delta_{n}\right)}\right)+\theta_{n, 3 s}, \\
& B_{t}=\sqrt{\left(B_{n+5,4 p}\right)^{2}+\left(B_{n, 3 s}\right)^{2}+2 B_{n+5,4 p} B_{n, 3 s} \cos \left(\delta_{n}\right),}
\end{aligned}
$$

with $\theta_{n, 3 s}=\Delta \phi_{n}^{\mathrm{XUV}}+\Delta \phi_{n, 3 s}^{\mathrm{A}}$. The relative phase between the $4 p$ and $3 s$ oscillations, $\delta_{n}$, is defined by

$$
\delta_{n}=\Delta \phi_{n+5}^{\mathrm{XUV}}-\Delta \phi_{n}^{\mathrm{XUV}}+\Delta \phi_{n+5,4 p}^{\mathrm{A}}-\Delta \phi_{n, 3 s}^{\mathrm{A}}+\pi,
$$

where the $\pi$ factor accounts for the opposite oscillations of $H_{n+5,4 p}$ with respect to the neighboring $4 p$ sidebands [46]. $\Delta \phi_{n+5}^{\mathrm{XUV}}-\Delta \phi_{n}^{\mathrm{XUV}} \approx 0.14 \pi$ is determined by an independent measurement of the attosecond chirp. The $3 \mathrm{~s}$ atomic phase is computed using the 2P2C-RPAE theory restricted to $n=3$ correlation, while the $4 p$ atomic phase is computed using the asymptotic approximation, as described in Ref. [46]. The resulting atomic delay for $4 p \mathrm{SU}$, shown in Fig. 4(b), is found to be small, due to cancellation of the positive $\Delta \phi_{n+5,4 p}^{\mathrm{W}}$ and negative $\Delta \phi_{n+5,4 p}^{\mathrm{cc}}$ contributions. This implies that the atomic

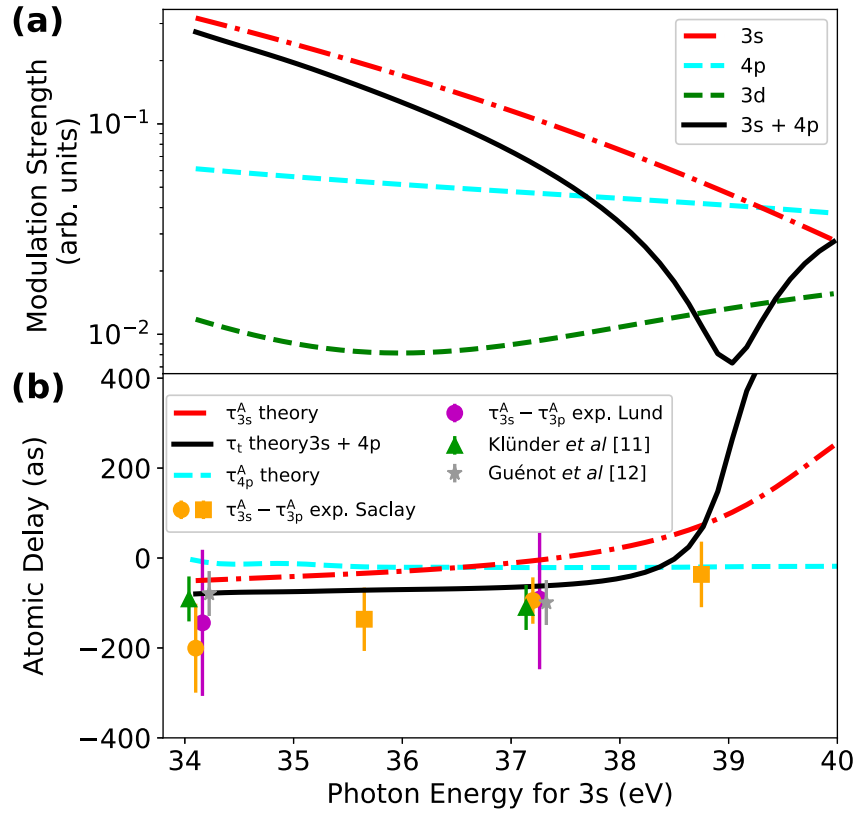

FIG. 4. (a) Estimated modulation strength $B_{n, i}$ of the RABBIT signal for the $3 s$ (red/medium gray line), $4 p$ (cyan/light gray line), and $3 d$ (green/dark gray line) channels, and effective modulation strength $B_{t}$ of the incoherently combined $3 s$ and $4 p$ contributions, denoted $3 s+4 p$ (black line). (b) Theoretical atomic delays for the $3 s$ (red/medium gray line) and $4 p$ (cyan/light gray line) channels, and effective atomic delay for the incoherent $3 s+4 p$ case (black line). The experimental points as well as the previously published data $[11,12]$ are plotted for comparison (in this region, $\tau_{3 p}^{\mathrm{A}} \sim 0$ ).

phase $\Delta \phi_{n+5,4 p}^{\mathrm{A}}$ plays a negligible role in Eq. (3). Similarly $\Delta \phi_{n, 3 s}^{\mathrm{A}}$, as shown in Fig. 3(b), is small so that $\delta_{n}$ is close but larger than $\pi$.

In the 34- to 38.5-eV range where $B_{n+5,4 p} \ll B_{n, 3 s}, \theta_{t} \approx$ $\left(\pi-\delta_{n}\right) B_{n+5,4 p} / B_{n, 3 s}+\theta_{n, 3 s}$. The contribution of the $4 p \mathrm{SU}$ channel shifts down the effective atomic delay, in excellent agreement with the experimental results in Fig. 4(b). Around $39 \mathrm{eV}$, the similar amplitudes and $\sim \pi$-shifted oscillations of the two channels result in a minimum of the total modulation strength $B_{t}$ and a fast variation of the effective delay as illustrated in Fig. 4 by the black lines. However, the $3 d$ SU channel cannot be neglected anymore in this energy region. This SU process is much more complicated to estimate because it originates from the highly correlated $3 s$ channel and exhibits a Cooper-like minimum as shown in Figs. 1(a) and 4(a). The 39- to $42-\mathrm{eV}$ range is thus a transition region where at least three dephased incoherent channels contribute. This might explain why the delay measured at harmonic 27 (close to $42 \mathrm{eV}$ ) cannot be easily reproduced by modeling.

In conclusion, we have demonstrated the potential of the Rainbow RABBIT method to separate the contributions of the $3 s$ and $3 p$ ionization channels in argon and to measure the corresponding atomic delays for a wide range of energies (34-68 eV) that include both $3 s$ and $3 p$ Cooper minima. Our results are in excellent agreement with the predictions of many-body perturbation theory in a $24-\mathrm{eV}$ range around the $3 p$ Cooper minimum, revealing the high accuracy of both experiment and theory in this region. Furthermore, we identify two strong shake-up ionization channels, the contributions of 
which are probably responsible for the discrepancy observed in the $3 s$ Cooper minimum region. This calls for further investigations to clarify their role. For instance, using a midIR driving wavelength from an optical parametric amplifier would allow for better sampling and tunability in order to separate the $3 s, 3 p$, and shake-up channels. This study thus provides a step towards improving our understanding of the complex nature of correlated multielectron ionization dynamics.

This research was supported by Agence Nationale de la Recherche, Grants No. ANR-15-CE30-0001-CIMBAAD,
No. ANR-11-EQPX0005-ATTOLAB, No. ANR-10-LABX0039-PALM; COST, Grant No. CA18222-AttoChem and Laserlab-Europe, Grant No. EU-H2020-654148. The authors affiliated in Sweden acknowledge support from the Swedish Research Council (Grants No. 2018-03845, No. 2013-08185, and No. 2016-03789) and the Knut and Alice Wallenberg Foundation (Grant No. 2017.0104). J.M.D. acknowledges support from the Swedish Foundation's Starting Grant by the Olle Engkvist's Foundation. The Lund group thanks Raimund Feifel and Richard J. Squibb, Gothenburg University, for lending their magnetic bottle electron spectrometer.
[1] L. Eisenbud, The formal properties of nuclear collisions, Ph.D. thesis, Princeton University, 1948.

[2] E. P. Wigner, Lower limit for the energy derivative of the scattering phase shift, Phys. Rev. 98, 145 (1955).

[3] F. T. Smith, Lifetime matrix in collision theory, Phys. Rev. 118, 349 (1960).

[4] A. L. Cavalieri, N. Müller, T. Uphues, V. S. Yakovlev, A. Baltuška, B. Horvath, B. Schmidt, L. Blümel, R. Holzwarth, S. Hendel, M. Drescher, U. Kleineberg, P. M. Echenique, R. Kienberger, F. Krausz, and U. Heinzmann, Attosecond spectroscopy in condensed matter, Nature (London) 449, 1029 (2007).

[5] M. Ossiander, J. Riemensberger, S. Neppl, M. Mittermair, M. Schäffer, A. Duensing, M. S. Wagner, R. Heider, M. Wurzer, M. Gerl, M. Schnitzenbaumer, J. V. Barth, F. Libisch, C. Lemell, J. Burgdörfer, P. Feulner, and R. Kienberger, Absolute timing of the photoelectric effect, Nature (London) 561, 374 (2018).

[6] S. Haessler, B. Fabre, J. Higuet, J. Caillat, T. Ruchon, P. Breger, B. Carré, E. Constant, A. Maquet, E. Mével, P. Salières, R. Taïeb, and Y. Mairesse, Phase-resolved attosecond nearthreshold photoionization of molecular nitrogen, Phys. Rev. A 80, 011404(R) (2009).

[7] M. Huppert, I. Jordan, D. Baykusheva, A. von Conta, and H. J. Wörner, Attosecond Delays in Molecular Photoionization, Phys. Rev. Lett. 117, 093001 (2016).

[8] S. Beaulieu, A. Comby, A. Clergerie, J. Caillat, D. Descamps, N. Dudovich, B. Fabre, R. Géneaux, F. Légaré, S. Petit, B. Pons, G. Porat, T. Ruchon, R. Taïeb, V. Blanchet, and Y. Mairesse, Attosecond-resolved photoionization of chiral molecules, Science 358, 1288 (2017).

[9] J. Vos, L. Cattaneo, S. Patchkovskii, T. Zimmermann, C. Cirelli, M. Lucchini, A. Kheifets, A. S. Landsman, and U. Keller, Orientation-dependent stereo Wigner time delay and electron localization in a small molecule, Science 360, 1326 (2018).

[10] M. Schultze, M. Fieß, N. Karpowicz, J. Gagnon, M. Korbman, M. Hofstetter, S. Neppl, A. L. Cavalieri, Y. Komninos, Th. Mercouris, C. A. Nicolaides, R. Pazourek, S. Nagele, J. Feist, J. Burgdörfer, A. M. Azzeer, R. Ernstorfer, R. Kienberger, U. Kleineberg, E. Goulielmakis et al., Delay in photoemission, Science 328, 1658 (2010).

[11] K. Klünder, J. M. Dahlström, M. Gisselbrecht, T. Fordell, M. Swoboda, D. Guénot, P. Johnsson, J. Caillat, J. Mauritsson, A. Maquet, R. Taïeb, and A. L'Huillier, Probing Single-Photon Ionization on the Attosecond Time Scale, Phys. Rev. Lett. 106, 143002 (2011).
[12] D. Guénot, K. Klünder, C. L. Arnold, D. Kroon, J. M. Dahlström, M. Miranda, T. Fordell, M. Gisselbrecht, P. Johnsson, J. Mauritsson, E. Lindroth, A. Maquet, R. Taïeb, A. L'Huillier, and A. S. Kheifets, Photoemission-time-delay measurements and calculations close to the $3 s$-ionizationcross-section minimum in Ar, Phys. Rev. A 85, 053424 (2012).

[13] D. Guénot, D. Kroon, E. Balogh, E. W. Larsen, M. Kotur, M. Miranda, T. Fordell, P. Johnsson, J. Mauritsson, M. Gisselbrecht, K. Varju, C. L. Arnold, T. Carette, A. S. Kheifets, E. Lindroth, A. L'Huillier, and J. M. Dahlström, Measurements of relative photoemission time delays in noble gas atoms, J. Phys. B: At., Mol. Opt. Phys. 47, 245602 (2014).

[14] C. Palatchi, J. M. Dahlström, A. S. Kheifets, I. A. Ivanov, D. M. Canaday, P. Agostini, and L. F. DiMauro, Atomic delay in helium, neon, argon and krypton, J. Phys. B: At., Mol. Opt. Phys. 47, 245003 (2014).

[15] M. Sabbar, S. Heuser, R. Boge, M. Lucchini, T. Carette, E. Lindroth, L. Gallmann, C. Cirelli, and U. Keller, Resonance Effects in Photoemission Time Delays, Phys. Rev. Lett. 115, 133001 (2015).

[16] S. Heuser, Á. Jiménez Galán, C. Cirelli, C. Marante, M. Sabbar, R. Boge, M. Lucchini, L. Gallmann, I. Ivanov, A. S. Kheifets, J. M. Dahlström, E. Lindroth, L. Argenti, F. Martín, and U. Keller, Angular dependence of photoemission time delay in helium, Phys. Rev. A 94, 063409 (2016).

[17] I. Jordan, M. Huppert, S. Pabst, A. S. Kheifets, D. Baykusheva, and H. J. Wörner, Spin-orbit delays in photoemission, Phys. Rev. A 95, 013404 (2017).

[18] M. Ossiander, F. Siegrist, V. Shirvanyan, R. Pazourek, A. Sommer, T. Latka, A. Guggenmos, S. Nagele, J. Feist, J. Burgdörfer, R. Kienberger, and M. Schultze, Attosecond correlation dynamics, Nat. Phys. 13, 280 (2017).

[19] M. Isinger, R. J. Squibb, D. Busto, S. Zhong, A. Harth, D. Kroon, S. Nandi, C. L. Arnold, M. Miranda, J. M. Dahlström, E. Lindroth, R. Feifel, M. Gisselbrecht, and A. L'Huillier, Photoionization in the time and frequency domain, Science $\mathbf{3 5 8}$, 893 (2017)

[20] C. Cirelli, C. Marante, S. Heuser, C. L. M. Petersson, Á Jiménez-Galán, L. Argenti, S. Zhong, D. Busto, M. Isinger, S. Nandi, S. Maclot, L. Rading, P. Johnsson, M. Gisselbrecht, M. Lucchini, L. Gallmann, J. M. Dahlström, E. Lindroth, A. L'Huillier, F. Martín, and U. Keller, Anisotropic photoemission time delays close to a Fano resonance, Nat. Commun. 9, 955 (2018). 
[21] J. Joseph, F. Holzmeier, D. Bresteau, C. Spezzani, T. Ruchon, J.-F. Hergott, O. Tcherbakoff, P. D’Oliveira, J. C. Houver, and D. Dowek, Angle-resolved studies of XUV-IR two-photon ionization in the RABBITT scheme, J. Phys. B: At., Mol. Opt. Phys. 53, 184007 (2020).

[22] H. Beutler, Über absorptionsserien von argon, krypton und xenon zu termen zwischen den beiden ionisierungsgrenzen ${ }^{2} P_{3}^{2 / 0}$ und ${ }^{2} P_{1}^{2 / 0}$, Z. Phys. 93, 177 (1935).

[23] U. Fano, Effects of configuration interaction on intensities and phase shifts, Phys. Rev. 124, 1866 (1961).

[24] J. L. Dehmer, Evidence of effective potential barriers in the xray absorption spectra of molecules, J. Chem. Phys. 56, 4496 (1972).

[25] J. W. Cooper, Photoionization from outer atomic subshells. A model study, Phys. Rev. 128, 681 (1962).

[26] M. Kotur, D. Guénot, Á. Jiménez-Galán, D. Kroon, E. W. Larsen, M. Louisy, S. Bengtsson, M. Miranda, J. Mauritsson, C. L. Arnold, S. E. Canton, M. Gisselbrecht, T. Carette, J. M. Dahlström, E. Lindroth, A. Maquet, L. Argenti, F. Martín, and A. L'Huillier, Spectral phase measurement of a Fano resonance using tunable attosecond pulses, Nat. Commun. 7, 10566 (2016).

[27] V. Gruson, L. Barreau, Á. Jiménez-Galan, F. Risoud, J. Caillat, A. Maquet, B. Carré, F. Lepetit, J.-F. Hergott, T. Ruchon, L. Argenti, R. Taïeb, F. Martín, and P. Salières, Attosecond dynamics through a Fano resonance: Monitoring the birth of a photoelectron, Science 354, 734 (2016).

[28] D. Busto, L. Barreau, M. Isinger, M. Turconi, C. Alexandridi, A. Harth, S. Zhong, R. J. Squibb, D. Kroon, S. Plogmaker, M. Miranda, Á. Jiménez-Galán, L. Argenti, C. L. Arnold, R. Feifel, F. Martín, M. Gisselbrecht, A. L'Huillier, and P. Salières, Timefrequency representation of autoionization dynamics in helium, J. Phys. B: At., Mol. Opt. Phys. 51, 044002 (2018).

[29] L. Barreau, C. L. M. Petersson, M. Klinker, A. Camper, C. Marante, T. Gorman, D. Kiesewetter, L. Argenti, P. Agostini, J. González-Vázquez, P. Salières, L. F. DiMauro, and F. Martín, Disentangling Spectral Phases of Interfering Autoionizing States from Attosecond Interferometric Measurements, Phys. Rev. Lett. 122, 253203 (2019).

[30] M. Turconi, L. Barreau, D. Busto, M. Isinger, C. Alexandridi, A. Harth, R. J. Squibb, D. Kroon, C. L. Arnold, R. Feifel, M. Gisselbrecht, L. Argenti, F. Martín, A. L'Huillier, and P. Salières, Spin-orbit-resolved spectral phase measurements around a Fano resonance, J. Phys. B: At., Mol. Opt. Phys. 53, 184003 (2020).

[31] S. B. Schoun, R. Chirla, J. Wheeler, C. Roedig, P. Agostini, L. F. DiMauro, K. J. Schafer, and M. B. Gaarde, Attosecond Pulse Shaping Around a Cooper Minimum, Phys. Rev. Lett. 112, 153001 (2014).

[32] T. Scarborough, T. Gorman, F. Mauger, P. Sándor, S. Khatri, M. Gaarde, K. Schafer, P. Agostini, and L. F. DiMauro, Full characterization of a molecular Cooper minimum using highharmonic spectroscopy, Appl. Sci. 8, 1129 (2018).

[33] A. F. Starace, Theory of atomic photoionization, in Handbuch der Physik, edited by W. Mehlhorn (Springer, Berlin, 1982), Vol. 31, p. 1.

[34] J. A. R. Samson and W. C. Stolte, Precision measurements of the total photoionization cross-sections of $\mathrm{He}, \mathrm{Ne}, \mathrm{Ar}$, $\mathrm{Kr}$, and Xe, J. Electron Spectrosc. Relat. Phenom. 123, 265 (2002).
[35] M. Y. Amusia, V. K. Ivanov, N. A. Cherepkov, and L. V. Chernysheva, Interference effects in photoionization of noble gas atoms outer s-subshells, Phys. Lett. A 40, 361 (1972).

[36] B. Möbus, B. Magel, K.-H. Schartner, B. Langer, U. Becker, M. Wildberger, and H. Schmoranzer, Measurements of absolute Ar $3 s$ photoionization cross sections, Phys. Rev. A 47, 3888 (1993).

[37] A. S. Kheifets, Time delay in valence-shell photoionization of noble-gas atoms, Phys. Rev. A 87, 063404 (2013).

[38] J. M. Dahlström, T. Carette, and E. Lindroth, Diagrammatic approach to attosecond delays in photoionization, Phys. Rev. A 86, 061402(R) (2012).

[39] J. M. Dahlström and E. Lindroth, Study of attosecond delays using perturbation diagrams and exterior complex scaling, J. Phys. B: At., Mol. Opt. Phys. 47, 124012 (2014).

[40] M. Magrakvelidze, M. E.-A. Madjet, G. Dixit, M. Ivanov, and H. S. Chakraborty, Attosecond time delay in valence photoionization and photorecombination of argon: A time-dependent local-density-approximation study, Phys. Rev. A 91, 063415 (2015).

[41] L.-W. Pi and A. S. Landsman, Attosecond time delay in photoionization of noble-gas and halogen atoms, Appl. Sci. 8, 322 (2018).

[42] A. W. Bray, F. Naseem, and A. S. Kheifets, Simulation of angular-resolved RABBITT measurements in noble-gas atoms, Phys. Rev. A 97, 063404 (2018).

[43] S. A. Sato, H. Hübener, A. Rubio, and U. De Giovannini, First-principles simulations for attosecond photoelectron spectroscopy based on time-dependent density functional theory, Eur. Phys. J. B 91, 126 (2018).

[44] J. Vinbladh, J. M. Dahlström, and E. Lindroth, Many-body calculations of two-photon, two-color matrix elements for attosecond delays, Phys. Rev. A 100, 043424 (2019).

[45] A. S. Kheifets, D. Toffoli, and P. Decleva, Angular dependent time delay near correlation induced Cooper minima, J. Phys. B: At., Mol. Opt. Phys. 53, 115201 (2020).

[46] See Supplemental Material at http://link.aps.org/supplemental/ 10.1103/PhysRevResearch.3.L012012 for details on the experimental and theoretical methods.

[47] W. Wijesundera and H. P. Kelly, Correlation satellites in the photoelectron spectrum of argon, Phys. Rev. A 39, 634 (1989).

[48] U. Becker, B. Langer, H. G. Kerkhoff, M. Kupsch, D. Szostak, R. Wehlitz, P. A. Heimann, S. H. Liu, D. W. Lindle, T. A. Ferrett, and D. A. Shirley, Observation of Many New Argon Valence Satellites Near Threshold, Phys. Rev. Lett. 60, 1490 (1988).

[49] Y. Mairesse, A. de Bohan, L. J. Frasinski, H. Merdji, L. C. Dinu, P. Monchicourt, P. Breger, M. Kovačev, R. Taïeb, B. Carré, H. G. Muller, P. Agostini, and P. Salières, Attosecond synchronization of high-harmonic soft x-rays, Science 302, 1540 (2003).

[50] J. M. Dahlström, D. Guénot, K. Klünder, M. Gisselbrecht, J. Mauritsson, A. L'Huillier, A. Maquet, and R. Taïeb, Theory of attosecond delays in laser-assisted photoionization, Chem. Phys. 414, 53 (2013).

[51] J. M. Dahlström, A. L'Huillier, and A. Maquet, Introduction to attosecond delays in photoionization, J. Phys. B: At., Mol. Opt. Phys. 45, 183001 (2012).

[52] C. Bourassin-Bouchet, L. Barreau, V. Gruson, J.-F. Hergott, F. Quéré, P. Salières, and T. Ruchon, Quantifying Decoherence in Attosecond Metrology, Phys. Rev. X 10, 031048 (2020). 